\title{
Development of a Nonlinear Probability of Collision Tool for the Earth Observing System
}

\author{
David P. McKinley ${ }^{1}$
}

The Earth Observing System (EOS) spacecraft Terra, Aqua, and Aura fly in constellation with several other spacecraft in 705-kilometer mean altitude sun-synchronous orbits. All three spacecraft are operated by the Earth Science Mission Operations (ESMO) Project at Goddard Space Flight Center (GSFC). In 2004, the ESMO project began assessing the probability of collision of the EOS spacecraft with other space objects. In addition to conjunctions with high relative velocities, the collision assessment method for the EOS spacecraft must address conjunctions with low relative velocities during potential collisions between constellation members. Probability of Collision algorithms that are based on assumptions of high relative velocities and linear relative trajectories are not suitable for these situations; therefore an algorithm for handling the nonlinear relative trajectories was developed. This paper describes this algorithm and presents results from its validation for operational use.

The probability of collision is typically calculated by integrating a Gaussian probability distribution over the volume swept out by a sphere representing the size of the space objects involved in the conjunction. This sphere is defined as the Hard Body Radius. With the assumption of linear relative trajectories, this volume is a cylinder, which translates into simple limits of integration for the probability calculation. For the case of nonlinear relative trajectories, the volume becomes a complex geometry. However, with an appropriate choice of coordinate systems, the new algorithm breaks down the complex geometry into a series of simple cylinders that have simple limits of integration. This nonlinear algorithm will be discussed in detail in the paper.

The nonlinear Probability of Collision algorithm was first verified by showing that, when used in high relative velocity cases, it yields similar answers to existing high relative velocity/linear relative trajectory algorithms. The comparison with the existing high velocity/linear theory will also be used to determine at what relative velocity the analysis should use the new nonlinear theory in place of the existing linear theory.

The nonlinear algorithm was also compared to a known exact solution for the probability of collision between two objects when the relative motion is strictly circular and the error covariance is spherically symmetric. Figure 1 shows preliminary results from this comparison by plotting the probabilities calculated from the new algorithm and those from the exact solution versus the Hard Body Radius to Covariance ratio. These results show about $5 \%$ error when the Hard Body Radius is equal to one half the spherical covariance magnitude.

The algorithm was then combined with a high fidelity orbit state and error covariance propagator into a useful tool for analyzing low relative velocity/nonlinear relative

\footnotetext{
'Mission Analyst, a.i. solutions, Inc., Lanham, Maryland, 20706, david.mckinley@ai-solutions.com
} 
trajectories. The high fidelity propagator is capable of using atmospheric drag, central body gravitational, solar radiation, and third body forces to provide accurate prediction of the relative trajectories and covariance evolution. The covariance propagator also includes a process noise model to ensure realistic evolutions of the error covariance. This paper will describe the integration of the nonlinear probability algorithm and the propagators into a useful collision assessment tool. Finally, a hypothetical case study involving a low relative velocity conjunction between members of the Earth Observation System constellation will be presented.

Figure 1: Comparison of Pe with Truth for Circular Relative Motion

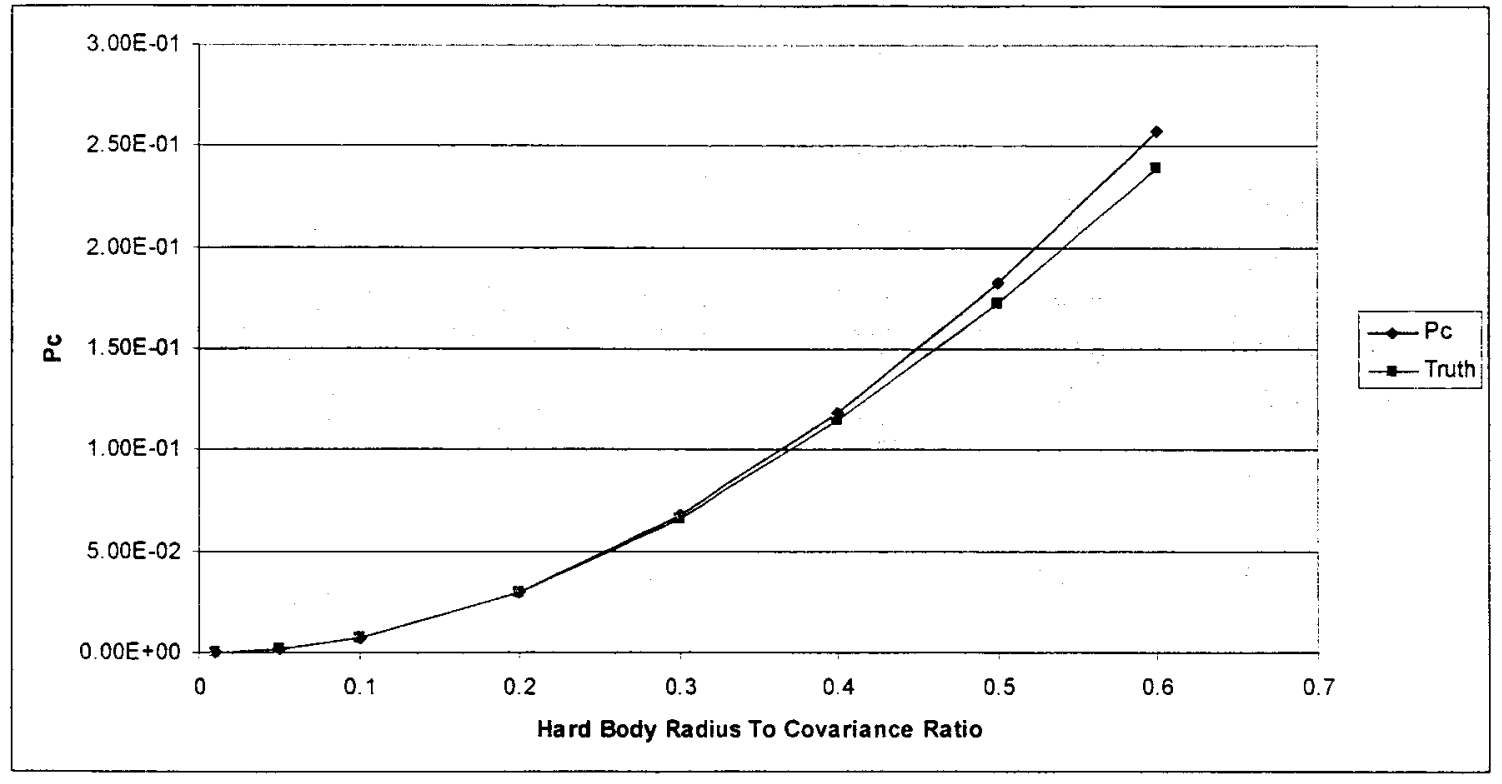




\title{
Development of a Nonlinear Probability of Collision Tool for the Earth Observing System
}

\author{
David P. McKinley \\ a.i. solutions, Inc. Lanham, MD, 20706
}

\begin{abstract}
The Earth Observing System began routine conjunction threat assessment of its member missions starting in 2004. It was quickly determined that a capability was needed to analyze conjunctions having nonlinear relative motion. An algorithm was developed that reduces the complex nonlinear relative motion into small linear segments that are easy to evaluate. The algorithm shows good agreement to the existing linear theory when used to analyze linear relative trajectories and for simple nonlinear cases for which solutions are known. The algorithm has been implemented in a software tool that accurately propagates the spacecraft states and covariances. The resulting 3D Collision Probability Tool is now being used by EOS analysts to study conjunction events.
\end{abstract}

\section{Introduction}

Starting in 2004, a collision risk identification and mitigation strategy was implemented for the Earth Observing System (EOS) series of spacecraft flown by Goddard Space Flight Center (GSFC). The 3 EOS spacecraft, Terra, Aqua, and Aura, fly with several other spacecraft in two constellations in $700 \mathrm{~km}$ mean equatorial altitude, sunsynchronous orbits. One of the key areas of interest was the capability to analyze and assess the collision risk during potential low relative velocity encounters between these spacecraft, since certain failure scenarios had been identified for the constellations that could lead to close approaches within a constellation [1]. Since the spacecraft in each constellation are in essentially the same orbital plane, it is reasonable to expect that interactions between the constellation members can be described as low relative velocity. Although existing tools compute a high relative velocity Probability of Collision $\left(\mathrm{P}_{\mathrm{c}}\right)$, the limits of applicability for that calculation as velocity transitions to a low relative velocity case are not well understood or documented. It was determined that a tool was needed that was capable of calculating the probability for low relative velocity encounters, not only so that low relative velocity encounters could be analyzed, but also so that an understanding of the transition from low relative velocity encounters to high relative velocity encounters could be analyzed. This paper documents the development of such a tool.

A great deal of work has been done on calculating $P_{c}$ for high relative velocity encounters $[2,3,4]$. The problem of low relative velocity has been investigated by Chan [5] and Patera [6]. Chan's method uses an analytical expression for the relative trajectory in order to derive the $P_{c}$. Patera's method uses a numerical scheme to evaluate the trajectory in increments to calculate the $\mathrm{P}_{c}$.

This paper describes the algorithm and tools that have been developed for EOS to handle the calculation of $P_{c}$ between two spacecraft whose relative trajectory is nonlinear. The algorithm developed is similar to that of Patera [6], although the reduction of the volume integral into a contour integral is bypassed because the limits of integration that arise from the algorithm are easily expressed in rectangular coordinates. The derivation of the algorithm is presented, and then it is shown that the new algorithm is equivalent to existing algorithms for high relative velocity cases. The use of the algorithm to evaluate the breakdown of the high relative velocity calculations is briefly. examined. Finally, a realistic case study is examined through implementation of the algorithm within the commercial off-the-shelf astrodynamics software package FreeFlyer ${ }^{\circledR}$. 


\section{Background}

The general structure of the probabilistic collision problem is shown in Figure 1. An object of concern, called the Primary, is predicted to come into close proximity with another object, called the Secondary. Without loss of generality, the Secondary's position is taken as the origin and the Primary is visualized as moving along the relative trajectory. The combined geometric extent of the Primary and Secondary is described by a sphere of radius $\mathrm{R}_{\mathrm{hb}}$, referred to as the combined Hard Body Radius (HBR). This sphere is attached to the Primary and sweeps out a volume, $\mathrm{V}$, during the time of interest. A 3-dimensional relative probability density function (PDF), $C(t)$, which is defined as the sum of Primary's and Secondary's position covariance matrices, is attached to Secondary. Generally, $\mathrm{C}(\mathrm{t})$ possesses a time-dependent shape and orientation due to the time variation in the covariance matrices of the separate objects.

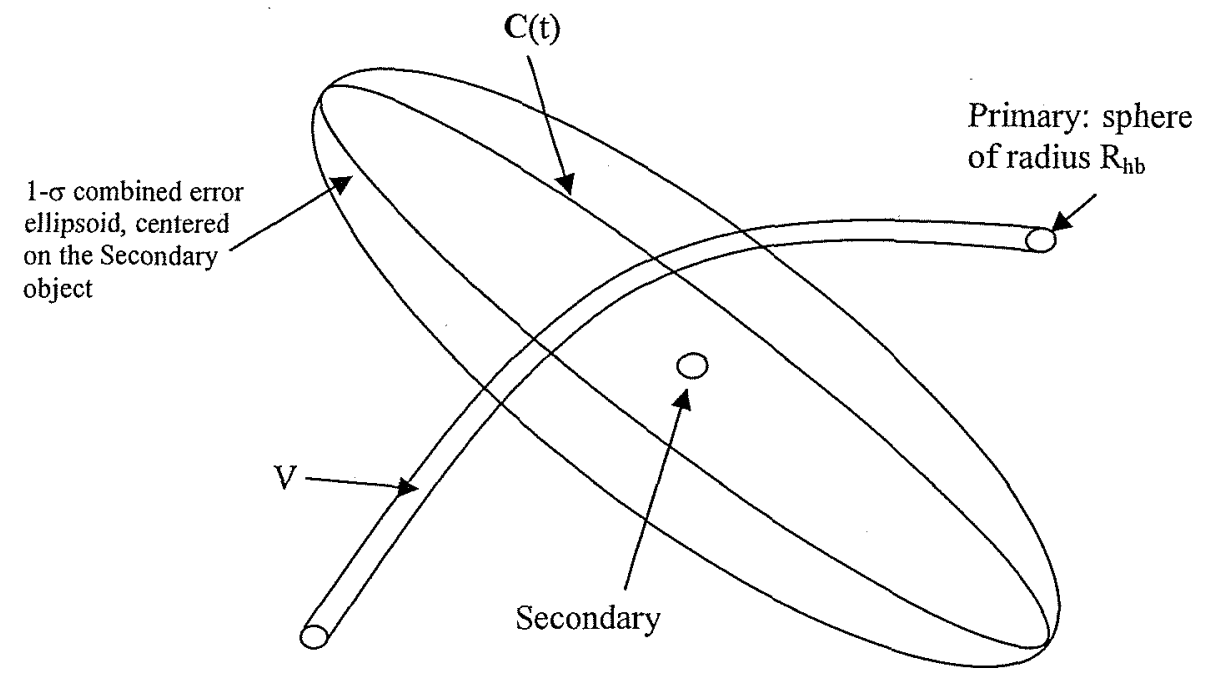

Figure 1: Typical Encounter Geometry.

The probability of collision, $\mathrm{P}_{\mathfrak{c}}$, is defined as the integral of the PDF over the swept-out volume V [3]:

$$
P_{c}=\frac{1}{\sqrt{(2 \pi)^{3}|\mathbf{C}|}} \iiint_{V} e^{-\frac{1}{2} \rho_{T} C^{-1} \rho} d X d Y d Z
$$

where $f=\left\{\begin{array}{l}x-x_{0} \\ y-y_{0} \\ z-z_{0}\end{array}\right\}$ is in some convenient set of coordinates. The vector $\mu_{0}=\left\{\begin{array}{lll}x_{0} & y_{0} & z_{0}\end{array}\right\}$ is the offset of the origin of the covariance from the center of the coordinate system. When the coordinate system and the covariance are centered on a common origin, the vector $F_{0}$ is zero.

In general the integral in Equation (1) is difficult to evaluate because of the time dependencies arising from the timevarying nature of $\mathbf{C}(\mathrm{t})$, and from the fact that different portions of $\mathbf{C}(\mathrm{t})$ fall within the Hard Body Radius at different times as the Primary evolves along the relative trajectory.

Computation of Equation (1) can be simplified on those occasions when the relative motion is nearly rectilinear; this approximation is valid when the relative velocity between the Primary and Secondary objects is high. This relative motion, called the linear or $2 \mathrm{D}$ case, allows the well-known reduction of Equation (1) to a two-dimensional integral $[2,3,4]$. Figure 2 demonstrates this linear configuration. A conjunction frame is constructed centered on the Secondary. The $\hat{x}$-axis of the frame points to the Primary at the time of closest approach (TCA). The $\hat{y}$-axis is 
along the direction of the relative velocity, and the $\hat{z}$-axis completes the right handed coordinate system. This construction allows for the long axis of the cylinder $V$ to be in the direction of the $\hat{y}$-axis of the conjunction frame.

Certain assumptions are made when solving the linear high relative velocity case. First, it is assumed that the encounter time is sufficiently small so that the individual covariance matrices and the resulting PDF are constant over the time of interest. Second, the high relative velocity means that the volume $\mathrm{V}$ will be several times longer than the 3-sigma value of the covariance in the $\hat{y}$-axis. This equates to $\mathrm{V}$ essentially extending from negative infinity to positive infinity relative to the size of the combined covariance along the $\hat{y}$-axis of the conjunction frame. Therefore, the integral along the $\hat{y}$-axis is taken to be unity, and the problem expressed in Equation (1) may then be reduced to a convenient integral of a two-dimensional Gaussian PDF over the area, A (Figure 2), formed by the intersection of $\mathrm{V}$ with the conjunction plane:

$$
P_{c}=\frac{1}{\sqrt{(2 \pi)^{2}\left|\mathbf{C}^{*}\right|}} \iint_{A} e^{-\frac{1}{2} \rho^{T} \mathbf{C}^{*-1} \rho} d X d Z
$$

where $P=\left\{\begin{array}{l}x \\ z\end{array}\right\}$ and $C^{*}$ is the 2-dimensional covariance matrix that results from projecting $\mathbf{C}(\mathrm{t})$ into the conjunction plane.

However, the high relative velocity assumptions used in the construction of Equation (2) make it impractical to use in cases where the relative motion is nonlinear. The correct procedure would be to fall back on Equation (1) for a nonlinear trajectory. However, the nonlinear trajectory would create a complex geometry for the volume $\mathrm{V}$, making integration difficult. In the next section an algorithm is presented that makes use of a series of simple geometries that approximate a nonlinear volume $\mathrm{V}$. 


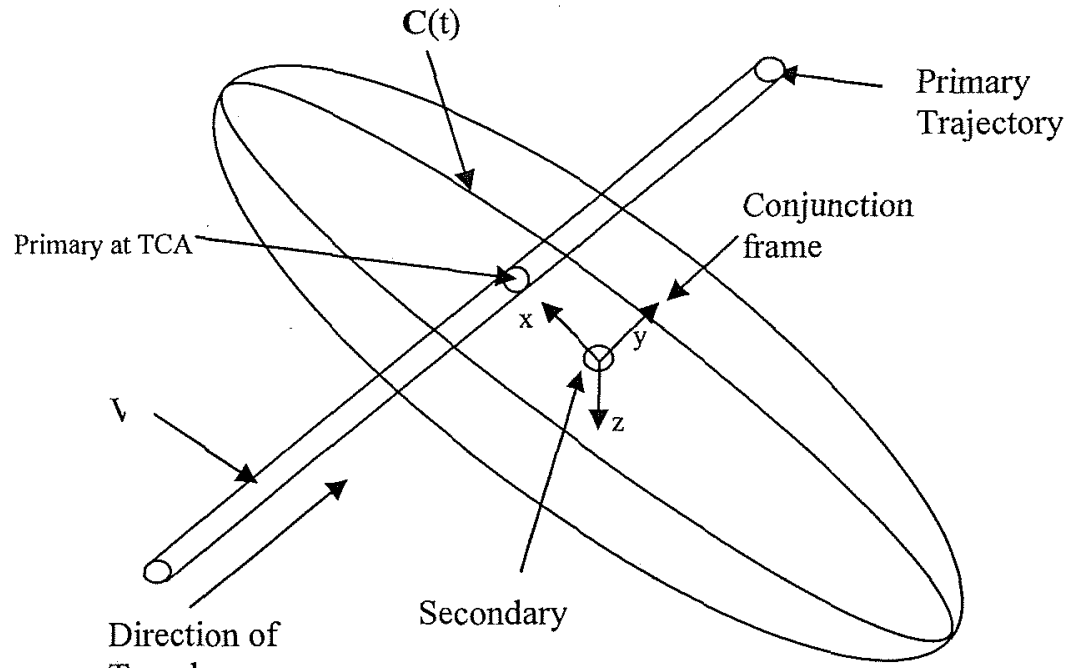

Travel

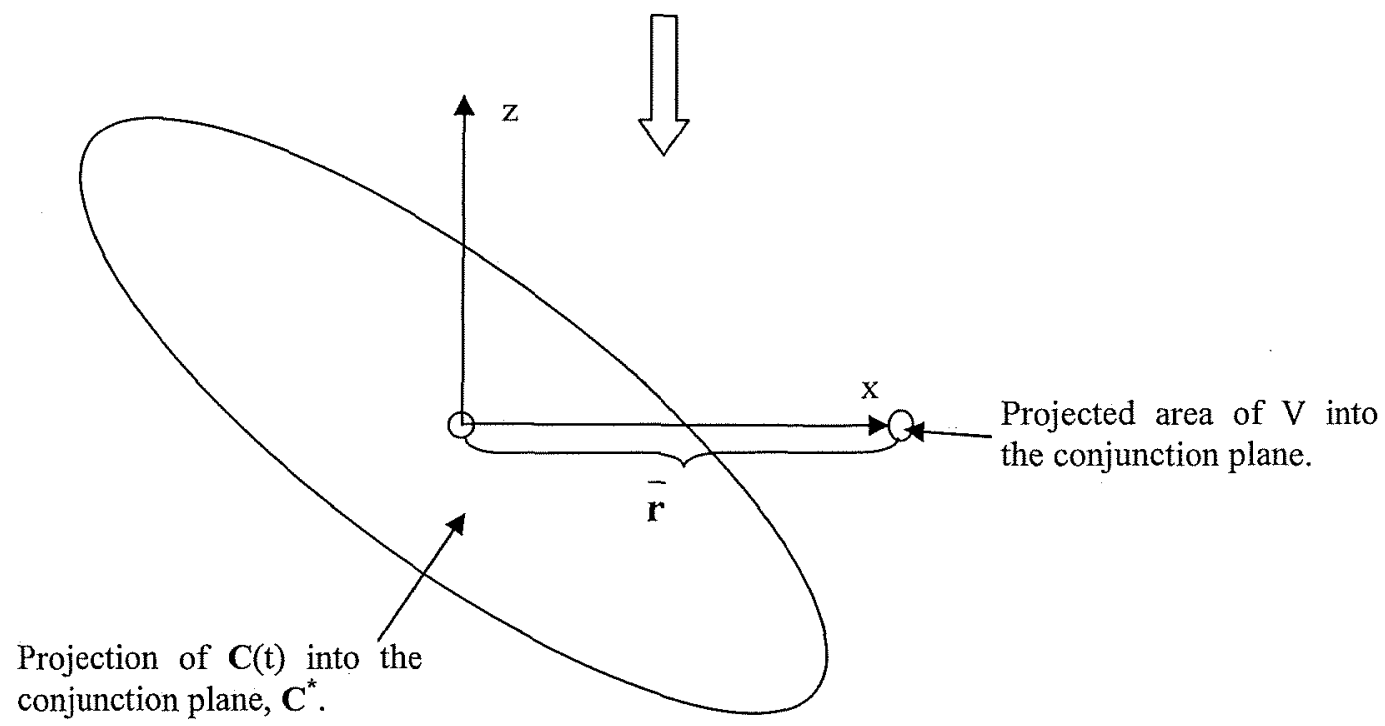

Figure 2: Reduction to two-dimensional integral for linear cases.

\section{Calculating the Probability of Collision along Nonlinear Relative Trajectory}

An algorithm similar to that given in Reference 6 has been implemented to investigate conjunctions with nonlinear relative trajectories. The algorithm differs from Reference 6 in that a new coordinate system in which to perform the numerical integration is developed. This limits of integration in the problem are easily defined in this new coordinate system, particularly when the problem of segment overlap is considered, as will be shown shortly.

To accommodate an arbitrary geometry, the volume $\mathrm{V}$ will be dissected into smaller, simpler geometries. Consider a sphere that defines the combined geometries of the Primary and Secondary objects with radius $R_{h b}$ as shown in Figure 3. During the encounter the sphere will similarly sweep out a long, curved, cylindrical volume. This volume can be thought of as made up of a series of smaller linear cylindrical volumes, $v_{i}$. The geometric centers of the edges of the smaller cylinders are defined by the relative position of the Primary object with respect to the Secondary object at the current time and at a time step in the future: $\vec{R}_{R E L_{i}}$ and $\vec{R}_{R E L_{i+1}}$, respectively. By making the 
time step between the two vectors small enough, the successive small linear cylinders can be made to approximate an arbitrary geometry defined by the nonlinear trajectory of the Primary relative to the Secondary.

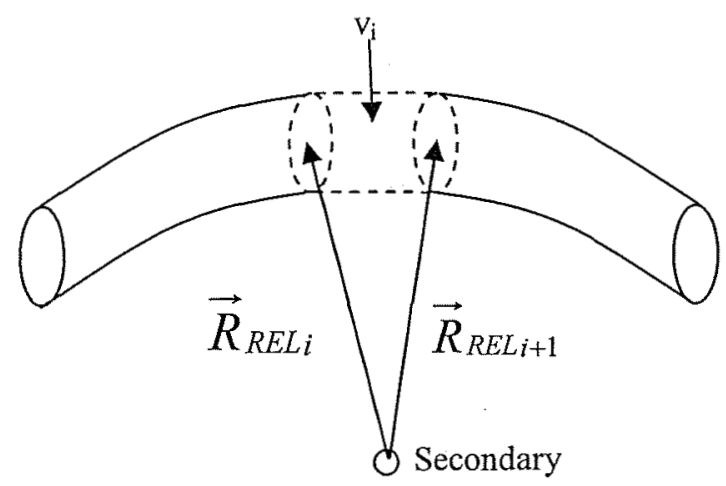

Figure 3: Dissection of complex geometry into small simple geometries.

The cylinders, $v_{i}$, can be used as the limits of integration in Equation (1). The total probability of collision for the encounter will then be the summation over all $\mathrm{v}_{\mathrm{i}}$. An additional advantage to this method is that the time steps may be sufficiently small such that the covariance is essentially constant over a single volume $v_{i}$. However, the covariance can change from its value at subsequent time steps.

The small simple geometries are easily integrated over using Equation (1) by constructing a convenient coordinate system at each time step, which is shown in Figure 4. The $\hat{V}_{i}$ direction is defined as the difference between the two relative positions $\hat{R}_{R E L_{i}}$ and $\hat{R}_{R E L_{i+1}}$. The $\hat{N}_{i}$ direction is defined as the normal to the plane containing $\hat{R}_{R E L_{i}}$ and $\hat{R}_{\dot{R E L} L_{i+1}}$. The $\hat{B}_{i}$ vector completes the right-handed system. The $\left\{\hat{V}_{i} \hat{N}_{i} \hat{B}_{i}\right\}$ system is centered on $k_{R E L_{i}}$ such that its origin does not coincide with the center of combined PDF.

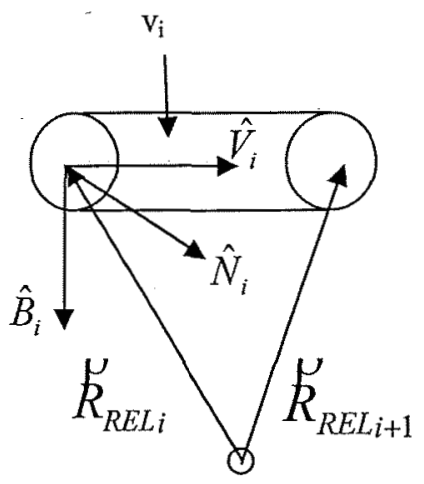

Figure 4: Definition of integration frame.

If the segments $v_{i}$ are left as right cylinders, there is the possibility of overlap between segments as shown in Figure 5. This overlap leads to integrating over the overlap volume twice, while not integrating over the excluded volume. When the HBR is large compared to the error covariance, this overlap issue can lead to incorrect probabilities [7]. To mitigate the overlap, each end of the cylinder $v_{i}$ is cut off at an angle defined by the angle the adjacent segments 
make to the current segment. These are angles $\theta_{1}$ and $\theta_{2}$ shown in Figure 6. There are four possibilities for the exact shape of each segment as shown in Figure 6 . The exact geometry can be determined by comparing the cross products $\hat{V}_{i} \times \hat{V}_{i-1}$ and $\hat{V}_{i+1} \times \hat{V}_{i}$ with the vector $\hat{N}_{i}$ as described in the algorithm section.

This method only accounts for overlap that occurs from nonlinearities in the in-plane relative motion. If there is a significant out-of-plane nonlinearity, i.e. $\hat{N}_{i}$ is significantly different from $\hat{N}_{i+1}$, then additional measures would need to be taken to eliminate this overlap. Patera [7] suggests one methodology for dealing with significant threedimensional overlap, and this topic remains one of the key areas for future work.

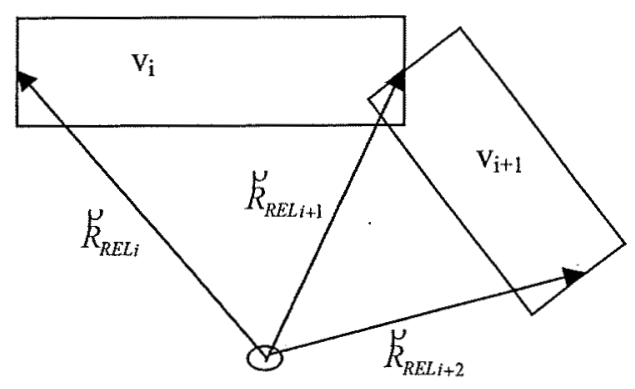

Figure 5. Overlap of cylindrical segments.
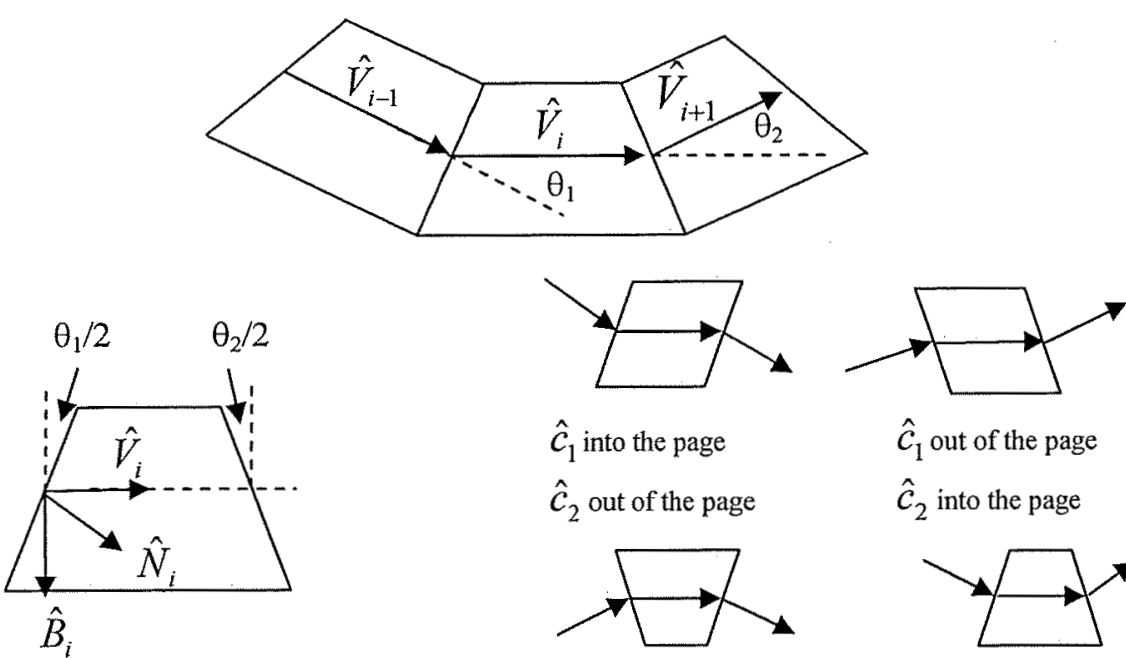

$\hat{c}_{2}$ out of the page

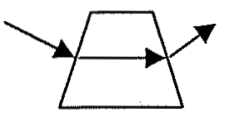

$$
\begin{array}{ll}
\hat{\mathcal{c}}_{1} \text { out of the page } & \hat{c}_{1} \text { into the page } \\
\hat{c}_{2} \text { out of the page } & \hat{\mathcal{c}}_{2} \text { into the page }
\end{array}
$$

Figure 6. Each segment $v_{i}$ is truncated at the end by the angle between the current segment and the adjacent segments. Four shapes are possible depending on the relative angles of the adjacent segments. 


\section{3D Algorithm}

An algorithm was developed based on the concepts described in Section III. The following steps take the Earth Centered Inertial (ECI) states and covariances for the Primary and Secondary objects and compute the probability of collision. The Primary and Secondary states and covariances are propagated to a particular time $i$, the integration frame is constructed, and the $P_{c_{i}}$ associated with that segment of the trajectory is computed. This process continues and the overall $\mathrm{P}_{\mathbf{c}}$ is the summation of the individual $P_{c_{i}}$.

1. The initial states and covariances at time i are labeled: ${ }^{J 2 K} R_{P i},{ }^{J 2 K} R_{S i},{ }^{J 2 K} \mathbf{C}_{P i},{ }^{J 2 K} \mathbf{C}_{S i}$. The subscript $\mathrm{P}$ represents state and covariance for the Primary object and $\mathrm{S}$ represents the secondary. Propagate the $\mathrm{J} 2000$ inertial states and covariance to time $\mathrm{i}+1$ and the inertial states also to time $\mathrm{i}+2$ to obtain ${ }^{J 2 K} k_{P_{i+1}}{ }^{J 2 K} K_{S_{i+1}}{ }^{J 2 K} \mathbf{C}_{P i+1},{ }^{J 2 K} \mathbf{C}_{S_{i+1}},{ }^{J 2 K} R_{P i+2},{ }^{J 2 K} k_{S_{i+2}}$.

2. Construct the transformation from $\mathrm{J} 2000$ to the integration frame at time $\mathrm{i}$ :

$$
\begin{aligned}
& { }^{J 2 K}{\stackrel{R}{R E L_{i+2}}}={ }^{J 2 K}{\stackrel{R}{S_{i+2}}}^{J 2 K} \breve{R}_{P_{i+2}} \\
& { }^{J 2 K} \hat{R}_{R E L_{i+1}}{ }^{J 2 K} \hat{R}_{S_{i+1}}-{ }^{J 2 K} K_{P_{i+1}} \\
& { }^{J 2 K} \hat{R}_{R E L_{i}}={ }^{J 2 K} \ddot{R}_{S_{i}}-{ }^{J 2 K} \ddot{R}_{P_{i}} \\
& \hat{V}_{i}=\left({ }^{52 K} \hat{R}_{R E L_{i+1}}-{ }^{J 2 K} \hat{R}_{R E L_{i}}\right)\|\|^{J 2 K} \hat{R}_{R E L_{i+1}}-{ }^{J 2 K} \hat{R}_{R E L_{i} \|} \| \\
& \hat{V}_{i+1}=\left({ }^{J 2 K} k_{R E L_{i+2}}-{ }^{J 2 K} R_{R E L_{i+1}}\right)\|\|^{J 2 K} \hat{R}_{R E L_{i+2}}-{ }^{J 2 K} R_{R E L_{i+1}} \|
\end{aligned}
$$

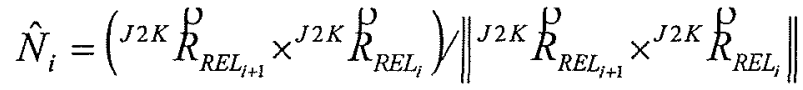

$$
\begin{aligned}
& \hat{B}_{i}=\hat{V}_{i} \times \hat{N}_{i} \\
& \mathbf{M}_{J 2 K_{i}}^{I N T}=\left[\begin{array}{ccc}
V_{x_{i}} & V_{y_{i}} & V_{z_{i}} \\
N_{x_{i}} & N_{y_{i}} & N_{z_{i}} \\
B_{x_{i}} & B_{y_{i}} & B_{z_{i}}
\end{array}\right]
\end{aligned}
$$

3. When the individual position uncertainty covariance matrices ${ }^{J 2 K} \mathbf{C}_{P_{i}}$ and ${ }^{J 2 K} \mathbf{C}_{S_{i}}$ are uncorrelated the uncertainty in the relative position between the primary and secondary object is described by the summation of the two matrices. Construct the combined covariance, transform it to the integration frame, and center it on the secondary object.

$$
{ }^{r e l} \mathbf{C}_{i}=\mathbf{M}_{J 2 K}^{I N T}\left({ }^{J 2 K} \mathbf{C}_{P_{i}}+{ }^{J 2 K} \mathbf{C}_{S i}\right) \mathbf{M}_{J 2 K}^{I N T}{ }^{T}
$$

4. Transform the miss distance vector from $\mathrm{J} 2000$ to the integration frame:

$$
{ }^{I N T} \ddot{R}_{R E L_{i}}=\mathbf{M}_{J 2 K}^{I N T}{ }^{J 2 K} \ddot{R}_{R E L_{i}}
$$

5. Compute the angles between adjacent segments $\theta_{1}$ and $\theta_{2}$ :

$$
\begin{aligned}
& \theta_{1}=\cos ^{-1}\left(\hat{V}_{i} \cdot \hat{V}_{i-1}\right) \\
& \theta_{2}=\cos ^{-1}\left(\hat{V}_{i+1} \cdot \hat{V}_{i}\right)
\end{aligned}
$$


6. Compute the vectors $\hat{c}_{1}$ and $\hat{c}_{2}$, which are used to determine the direction the trajectory is turning from segment to segment:

$$
\begin{aligned}
& \hat{c}_{1}=\left(\hat{V}_{i} \times \hat{V}_{i-1}\right) \\
& \hat{c}_{2}=\left(\hat{V}_{i+1} \times \hat{V}_{i}\right)
\end{aligned}
$$

7. By comparing the dot products $\hat{c}_{1} \cdot \hat{N}_{i}$ and $\hat{c}_{2} \cdot \hat{N}_{i}$, the direction the trajectory is turning is known, and the limits of integration can be defined. The variables of integration in the $\left\{\hat{V}_{i} \hat{N}_{i} \hat{B}_{i}\right\}$ system are $v, n$, and $b$. The limits for the $v$ integral are given as a function of $b$ and $\theta_{1}$ and $\theta_{2}$ :

If $\hat{c}_{1} \cdot \hat{N}_{i} \geq 0$ and $\hat{c}_{2} \cdot \hat{N}_{i}<0$

$$
b \tan \left(\frac{\theta_{1}}{2}\right) \leq y<\left\|J 2 K R_{R E L_{i+1}}-{ }^{J 2 K} R_{R E L_{i}}\right\|+b \tan \left(\frac{\theta_{2}}{2}\right)
$$

If $\hat{c}_{1} \cdot \hat{N}_{i}<0$ and $\hat{c}_{2} \cdot \hat{N}_{i}<0$

$$
-b \tan \left(\frac{\theta_{1}}{2}\right) \leq v<\left\|J 2 K{ }_{R E L_{i+1}}-{ }^{J 2 K} R_{R E L_{i}}\right\|+b \tan \left(\frac{\theta_{2}}{2}\right)
$$

If $\hat{c}_{1} \cdot \hat{N}_{i} \geq 0$ and $\hat{c}_{2} \cdot \hat{N}_{i} \geq 0$

$$
b \tan \left(\frac{\theta_{1}}{2}\right) \leq v<\left\|J 2 K \rho_{R E L_{i+1}}-{ }^{J 2 K} \rho_{R E L_{i}}\right\|-b \tan \left(\frac{\theta_{2}}{2}\right)
$$

If $\hat{c}_{1} \cdot \hat{N}_{i}<0$ and $\hat{c}_{2} \cdot \hat{N}_{i} \geq 0$

$$
-b \tan \left(\frac{\theta_{1}}{2}\right) \leq v<\left\|J 2 K \rho_{R E L_{i+1}}-{ }^{J 2 K} \rho_{R E L_{i}}\right\|-b \tan \left(\frac{\theta_{2}}{2}\right)
$$

8. Compute the limits of integration in the $\hat{N}_{i}$ and $\hat{B}_{i}$ directions:

$$
\begin{gathered}
-\sqrt{H B R^{2}-b^{2}} \leq n \leq \sqrt{H B R^{2}-b^{2}} \\
-H B R \leq b \leq H B R
\end{gathered}
$$

9. Integrate the Gaussian distribution over the segment $v_{i}$ to determine the incremental probability associated with step $\mathrm{i}$, where $v_{i}, n_{i}$ and $b_{i}$ are the components of the negative of the relative position vector: $-{ }^{I N T} K_{R E L_{i}}$

$$
P_{c_{i}}=\frac{1}{\sqrt{(2 \pi)^{3}\left|{ }^{\text {INT }} \mathbf{C}_{i}\right|}} \iiint_{v_{j}} e^{-\frac{1}{2} \rho_{T^{T} \text { int }} \mathbf{c}_{i}^{-1 \rho}} d V d N d B
$$




$$
\rho=\left\{\begin{array}{l}
v-v_{i} \\
n-n_{i} \\
b-b_{i}
\end{array}\right\}
$$

10. Save $\hat{R}_{i-1}=\hat{R}_{i}$. Return to Step 3 and repeat for the next time step until probability or trajectory conditions dictate that the loop ends. Discussion of when to terminate the loop is left to the following sections in which examples are given.

11. Accumulate the probability:

$$
P_{c}=\sum_{i} P_{c_{i}}
$$

\section{Comparison with Two-Dimensional Integration}

The algorithm presented in Equations (3) through (25), which will be referred to as the 3D method, makes no assumptions that would limit its use in linear as well as nonlinear relative trajectories. Therefore, using a linear relative trajectory, the probability can be computed using both the $3 \mathrm{D}$ method and the $2 \mathrm{D}$ method described in Equation (2). Simulated primary and secondary trajectories with 97-minute periods were generated with the Secondary object 500 meters directly below the Primary object in the radial direction at TCA. The angle between the two orbit planes was adjusted from 0 to 90 degrees to vary the relative velocity. A constant combined spherical position error covariance of $2 \mathrm{~km}^{2}$ was used, and the probability of collision was calculated.

Table 1 shows the results of the comparison of the 2D and 3D methods. As the angle between the two orbital planes decreases, the relative velocity at TCA decreases. For relative velocities as low as $13 \mathrm{~m} / \mathrm{s}$, the percent difference between the $3 \mathrm{D}$ and $2 \mathrm{D}$ probabilities is less than 1 percent, indicating good agreement between the 2D and 3D methods. As the relative velocities fall below about $2 \mathrm{~m} / \mathrm{s}$, the percent error grows quickly with the $2 \mathrm{D}$ method often giving probabilities an order of magnitude too conservative.

\begin{tabular}{|c|c|c|c|c|}
\hline $\begin{array}{c}\text { Orbit Plane } \\
\text { Angle } \\
\text { (degrees) }\end{array}$ & $\begin{array}{c}\text { Relative } \\
\text { Velocity }(\mathrm{m} / \mathrm{s})\end{array}$ & $\mathbf{P}_{\mathbf{c}}$ 3D & $\mathbf{P}_{\mathbf{c}}$ 2D & $\begin{array}{c}\text { Percent } \\
\text { Difference }\end{array}$ \\
\hline 90.00 & 10672.000 & $9.393687 \mathrm{E}-05$ & $9.393670 \mathrm{E}-05$ & $1.809729 \mathrm{E}-04$ \\
\hline 45.00 & 5775.000 & $9.330270 \mathrm{E}-05$ & $9.393690 \mathrm{E}-05$ & $6.751356 \mathrm{E}-01$ \\
\hline 10.00 & 1315.000 & $9.409870 \mathrm{E}-05$ & $9.393670 \mathrm{E}-05$ & $1.724566 \mathrm{E}-01$ \\
\hline 1.00 & 131.700 & $9.410713 \mathrm{E}-05$ & $9.393670 \mathrm{E}-05$ & $1.814288 \mathrm{E}-01$ \\
\hline 0.10 & 13.175 & $9.394736 \mathrm{E}-05$ & $9.393670 \mathrm{E}-05$ & $1.134807 \mathrm{E}-02$ \\
\hline 0.01 & 1.318 & $1.644402 \mathrm{E}-04$ & $9.393670 \mathrm{E}-05$ & $7.505425 \mathrm{E}+01$ \\
\hline 0.00 & 0.270 & $1.489750 \mathrm{E}-04$ & $9.393670 \mathrm{E}-05$ & $5.859084 \mathrm{E}+01$ \\
\hline
\end{tabular}

Table 1: Comparison of 2D and 3D methods for relative trajectories with various relative velocities.

The breakdown of the assumptions of the $2 \mathrm{D}$ method in this case happens below $13 \mathrm{~m} / \mathrm{s}$. Figure 7 shows the accumulated probability for the $5775 \mathrm{~m} / \mathrm{s}$ and $0.27 \mathrm{~m} / \mathrm{s}$ relative velocity cases. In the $5775 \mathrm{~m} / \mathrm{s}$ case, the total Probability of Collision is "accumulated" in under two seconds. In the $0.27 \mathrm{~m} / \mathrm{s}$ relative velocity case it takes nearly five hours for the probability to "accumulate". The long dwell time of the $0.27 \mathrm{~m} / \mathrm{s}$ case demonstrates the necessity of using a nonlinear algorithm to assess this probability.

A general statement on the breakdown of the two-dimensional assumptions should not be inferred from this simple example. The failure of the two-dimensional assumptions is possibly dependent on the covariance size, shape (most 
covariances are actually elliptical, not circular), and orientation. Further analysis is required to fully assess the transition between the two- and three-dimensional cases. Clearly, however, a breakdown of the two-dimensional case will occur, and a three dimensional tool is required to evaluate the collision probability in these cases.
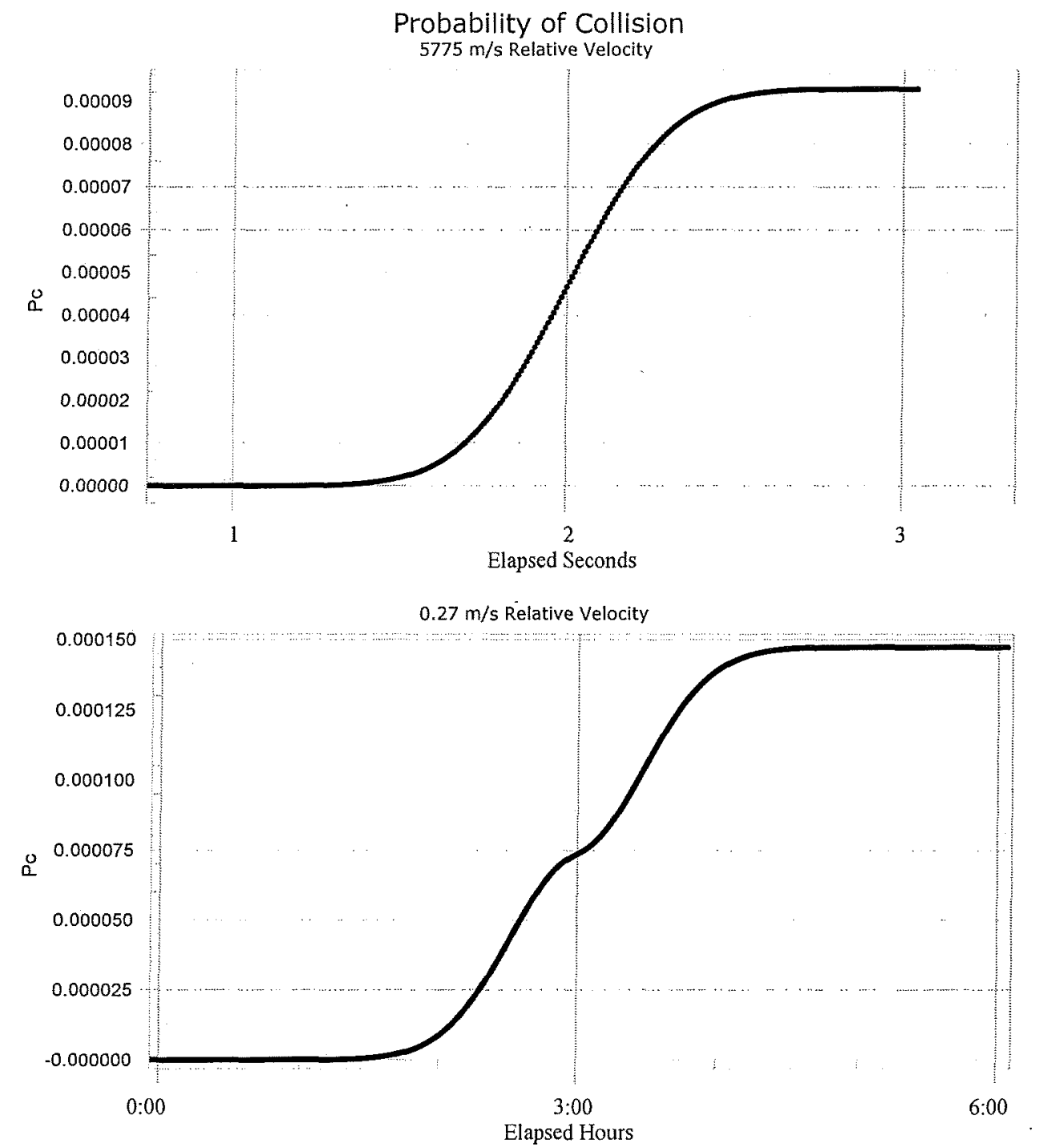

Figure 7. Accumulation of the Probability of Collision for low and high relative velocities using 3D method.

The $0.27 \mathrm{~m} / \mathrm{s}$ relative trajectory depicted in Figure 7 is nonlinear, but is also essentially a fly-by trajectory. In fly-by trajectories one object approaches the vicinity of the other, reaches a minimum miss distance, and then departs the vicinity. In fly-by cases the conditions under which the integration loop can be terminated are clear. When the distance between the objects is large compared to the combined covariance the contribution of the trajectory segment $v_{j}$ to the overall $P_{c}$ is negligible. Therefore, after the objects have left the vicinity of one another the probability no longer accumulates and the $P_{c}$ for this fly-by event is determined. Figure 7 then should not be interpreted as a time history of the $P_{c}$; rather it shows the summation of Equation 25 as the relative trajectory progresses. The $P_{c}$ must be tied to an event or time span, in this case a fly-by. The correct interpretation of Figure 7 is that the steady state value of $1.489 \mathrm{E}-04$ shown is the $P_{c}$ for the given fly-by event. 


\section{Nonlinear Trajectory}

A solution for the Probability of Collision has been given in Reference 6 for the case of a spherical hard body of radius $r$ in a circular relative trajectory about the Secondary object with a spherically symmetric covariance with uncertainty $\sigma$. The hard body radius centered on the Primary object sweeps out a torus of radius $R$ about the Secondary as shown in Figure 8. The truth probability for this case is given by Patera [6]:

$$
P_{c_{\text {truth }}}=\frac{2}{\sigma} \sqrt{\frac{2}{\pi}} \exp \left(\frac{-\left(R^{2}+H B R^{2}\right)}{2 \sigma^{2}}\right) \int \sinh \left(\frac{R \sqrt{H B R^{2}-x^{2}}}{\sigma^{2}}\right) d x .
$$

Chan [5] also derives a closed-form approximate solution for this case by assuming relative motion described by the Clohessey-Wiltshire equations. This approximation is given by:

$$
P_{c}=\sqrt{2 \pi} e^{-\left(R^{2}+H B R\right) / 2 \sigma^{2}} \frac{R \cdot H B R^{2}}{2 \sigma^{3}} .
$$

Equation (26) was numerically integrated and compared to results from Equation (27) and the new $3 \mathrm{D}$ algorithm. The simulation parameters were set to $\mathrm{R}=1 \mathrm{~km}$ and $\sigma=1 \mathrm{~km}$. A one-degree step size was used around the torus. In this case the $3 \mathrm{D}$ algorithm was terminated after the primary object completed 360 degrees, or one relative orbit. The resulting $P_{c}$ is therefore the $P_{c}$ for the event defined as one orbit of the primary object about the secondary. The resulting probabilities for various values of hard body radius to uncertainty ratios $(\mathrm{HBR} / \sigma)$ are shown in Table 2 , and the percent differences are shown in Figure 9. The results show good agreement between the 3D method and the existing methods, with the 3D method differing by less than $1 \%$ from the exact solution of Equation (26).

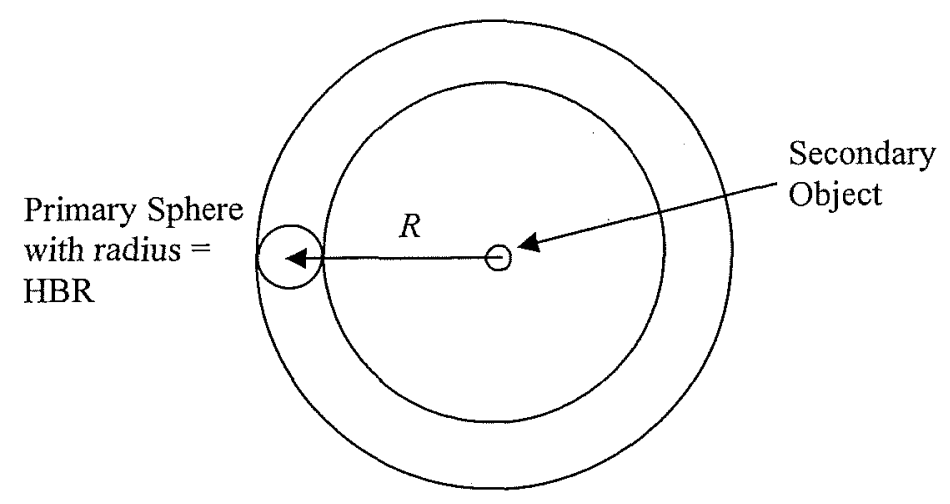

Figure 8: Circular Relative Motion of Primary about the Secondary. 


\begin{tabular}{|c|c|c|c|}
\hline $\begin{array}{c}\text { HBR to } \\
\text { Sigma Ratio }\end{array}$ & 3D & Patera (Eq. 26) & Chan (Eq. 27) \\
\hline 0.01 & $7.51446 \mathrm{E}-05$ & $7.58412 \mathrm{E}-05$ & $7.60135 \mathrm{E}-05$ \\
\hline 0.05 & $1.87901 \mathrm{E}-03$ & $1.89865 \mathrm{E}-03$ & $1.89805 \mathrm{E}-03$ \\
\hline 0.1 & $7.55945 \mathrm{E}-03$ & $7.57328 \mathrm{E}-03$ & $7.56382 \mathrm{E}-03$ \\
\hline 0.2 & $2.96574 \mathrm{E}-02$ & $2.99541 \mathrm{E}-02$ & $2.98048 \mathrm{E}-02$ \\
\hline 0.3 & $6.58325 \mathrm{E}-02$ & $6.61437 \mathrm{E}-02$ & $6.54051 \mathrm{E}-02$ \\
\hline 0.4 & $1.14105 \mathrm{E}-01$ & $1.14537 \mathrm{E}-01$ & $1.12276 \mathrm{E}-01$ \\
\hline 0.5 & $1.72380 \mathrm{E}-01$ & $1.73008 \mathrm{E}-01$ & $1.67712 \mathrm{E}-01$ \\
\hline 0.6 & $2.38647 \mathrm{E}-01$ & $2.39024 \mathrm{E}-01$ & $2.28582 \mathrm{E}-01$ \\
\hline 0.7 & $3.09401 \mathrm{E}-01$ & $3.09771 \mathrm{E}-01$ & $2.91546 \mathrm{E}-01$ \\
\hline 0.8 & $3.82735 \mathrm{E}-01$ & $3.82306 \mathrm{E}-01$ & $3.53280 \mathrm{E}-01$ \\
\hline 0.9 & $4.53239 \mathrm{E}-01$ & $4.53694 \mathrm{E}-01$ & $4.10685 \mathrm{E}-01$ \\
\hline 1 & $5.20980 \mathrm{E}-01$ & $5.21154 \mathrm{E}-01$ & $4.61069 \mathrm{E}-01$ \\
\hline
\end{tabular}

Table 2. Comparison of 3D method with Patera's exact solution and Chan's approximation.

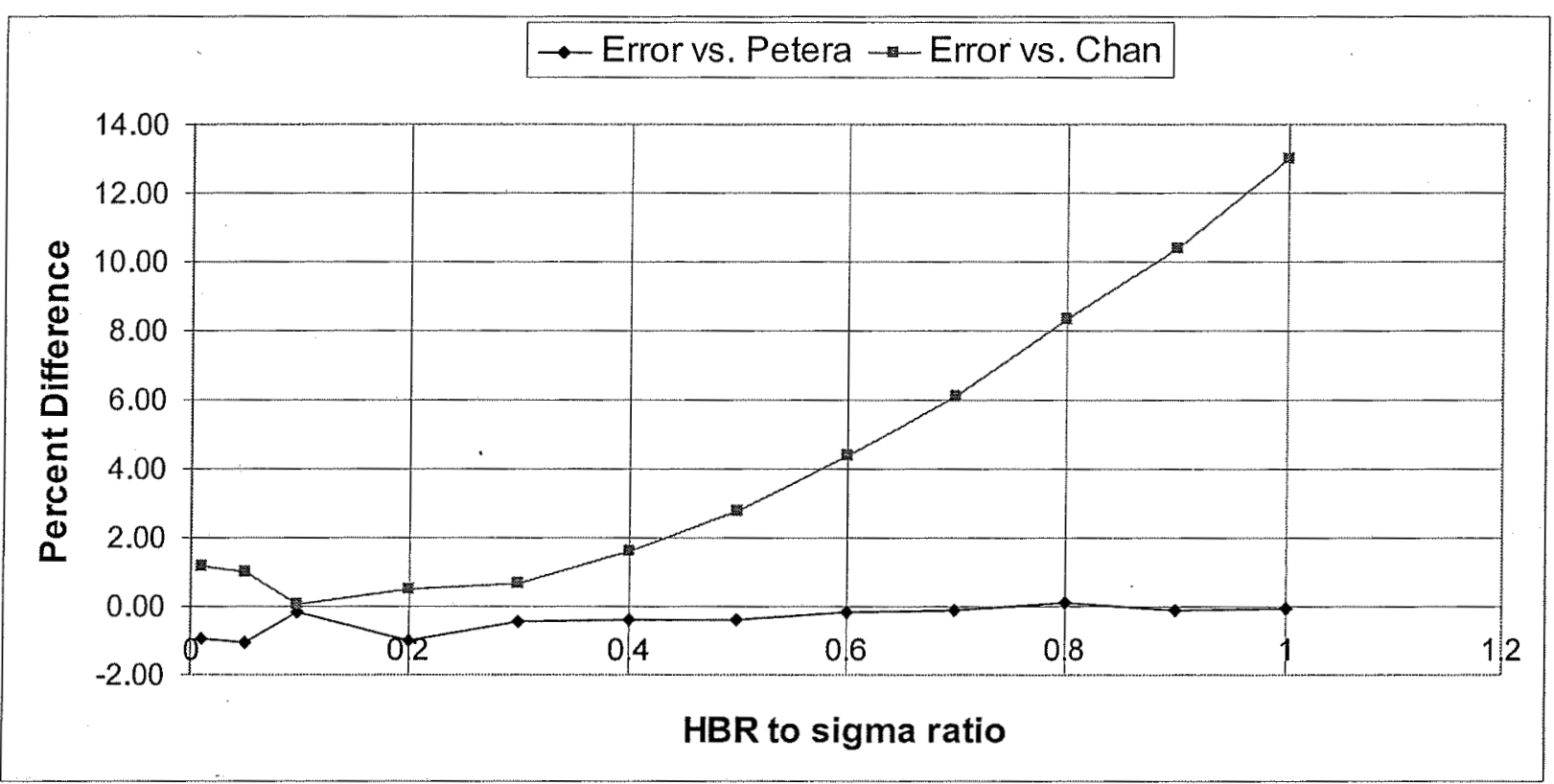

Figure 9. Percent difference between $\mathrm{P}_{c}$ calculated using 3D method, Patera's exact solution, and Chan's approximation.

\section{Case Study}

The 3D algorithm presented above was incorporated into the Conjunction Assessment and Mitigation (CAM) Tool Suite System, which was designed and built to support conjunction risk assessment and mitigation for EOS. The CAM Tool Suite was developed using the commercial off-the-shelf astrodynamics software FreeFlyer ${ }^{\circledR}$ (FF) and MATLAB ${ }^{\circledR}$. FF provides the means to propagate the states and covariances using a wide set of force model options. Additionally, FF provides a built-in interface to MATLAB ${ }^{\circledR}$, which is used to perform the numerical integration to determine the $\mathrm{P}_{\mathrm{c}}$.

A simulation of a hypothetical interaction between constellation members was developed. In this scenario, the Secondary object is slowly flying above the Primary object, and the relative motion is coplanar. At TCA the Secondary is approximately $50 \mathrm{~m}$ above the Primary and the relative velocity is approximately $1 \mathrm{~m} / \mathrm{s}$ for the entire 
encounter. Table 3 lists the orbital elements at TCA, and Table 4 lists the force model parameters used. The nonlinear relative trajectory in the Radial - In Track plane can be seen in Figure 10. The same initial covariance was used for each object in the Radial - In Track - Cross Track coordinate system:

$$
C=\left[\begin{array}{cccccc}
3 & 0 & 0 & 0 & -0.010989 & 0 \\
0 & 34 & 0 & -1.181196 & 0 & 0 \\
0 & 0 & 16 & 0 & 0 & 0 \\
0 & -1.181196 & 0 & 0.04 & 0 & 0 \\
-0.010989 & 0 & 0 & 0 & 0.004 & 0 \\
0 & 0 & 0 & 0 & 0 & 0.02
\end{array}\right]
$$

The time history of the Primary and Secondary object's covariance can be seen in Figures 11 and 12. Figure 13 shows that the $P_{c}$ computed for this encounter using the 3D method is 0.38877 . Although this relative trajectory has a significant nonlinear component, this is again a fly-by trajectory and Figure 13 shows the summation of Equation 25. The $P_{c}$ reported is the steady state value and represents the $P_{c}$ for this fly-by event.

To substantiate these results, a Monte Carlo simulation was also performed. The same states, covariances, and force models were used as inputs. For each Monte Carlo trial, the Primary and Secondary states were randomly sampled according to their covariance matrices. The sampled states were then propagated forward in time. If the range between the states went below the HBR of $20 \mathrm{~m}$, a conjunction was said to have occurred. The number of conjunctions divided by the number of trials was the resulting $\mathrm{P}_{\mathrm{c}}$. Figure 14 shows the results of the Monte Carlo simulation. The $\mathrm{P}_{\mathrm{c}}$ computed by Monte Carlo was between 0.125 and 0.15 , which is of a comparable magnitude to the results given by the $3 \mathrm{D}$ algorithm. This discrepancy is still under investigation.

\begin{tabular}{|c|c|c|}
\hline Parameter & Primary & Secondary \\
\hline $\mathrm{a}, \mathrm{km}$ & 7077 & 7078.8966 \\
\hline $\mathrm{e}$ & 0 & 0.0002665 \\
\hline i, degrees & 98 & 98 \\
\hline$\Omega$, degrees & 221 & 221 \\
\hline w, degrees & 90 & 90 \\
\hline $\mathrm{v}$, degrees & 0 & 0 \\
\hline
\end{tabular}

Table 3. Primary and Secondary orbital elements at TCA

\begin{tabular}{|l|l|l|}
\hline Parameter & Primary & Secondary \\
\hline Mass, kg & 3239 & 897 \\
\hline Geopotential & EGM96 4x4 & EGM96 4x4 \\
\hline Cd & 2.2 & 2.2 \\
\hline Drag Area & 47.95 & 8.3 \\
\hline Atmosphere & Jacchia Roberts & Jacchia Roberts \\
\hline $3^{\text {rd } \text { Body }}$ & Sun, Moon & Sun, Moon \\
\hline
\end{tabular}

Table 4. Force model properties for case study. 


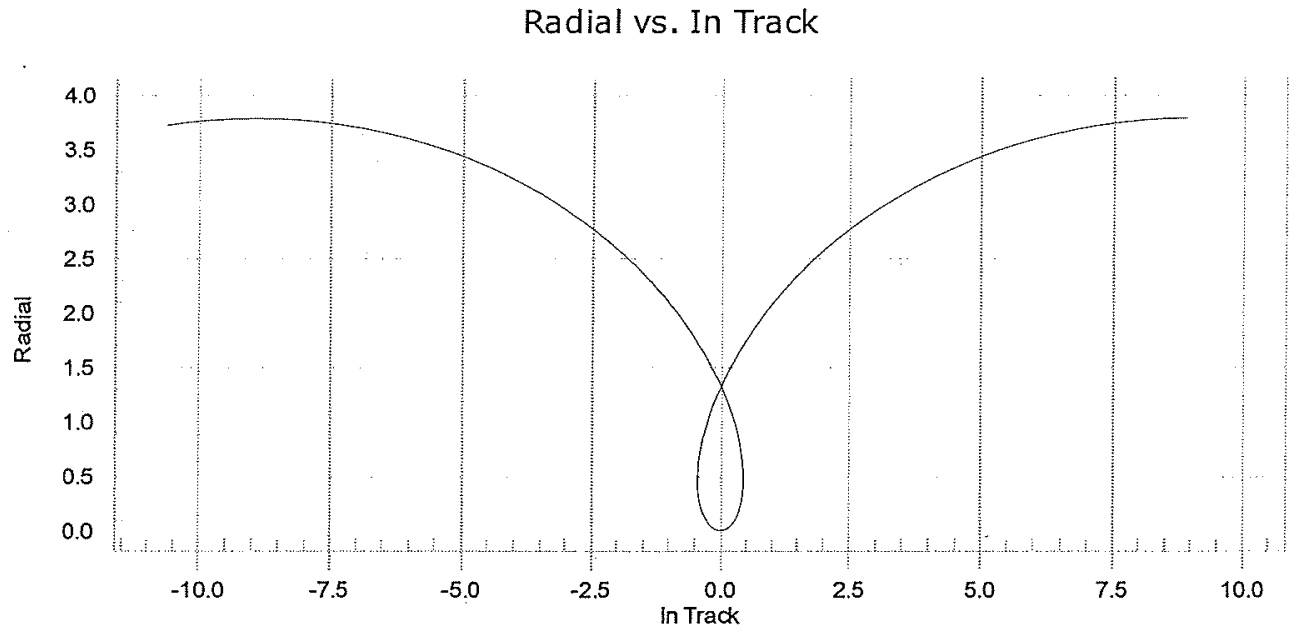

Figure 10. Nonlinear relative trajectory of the Secondary object relative to the Primary object.

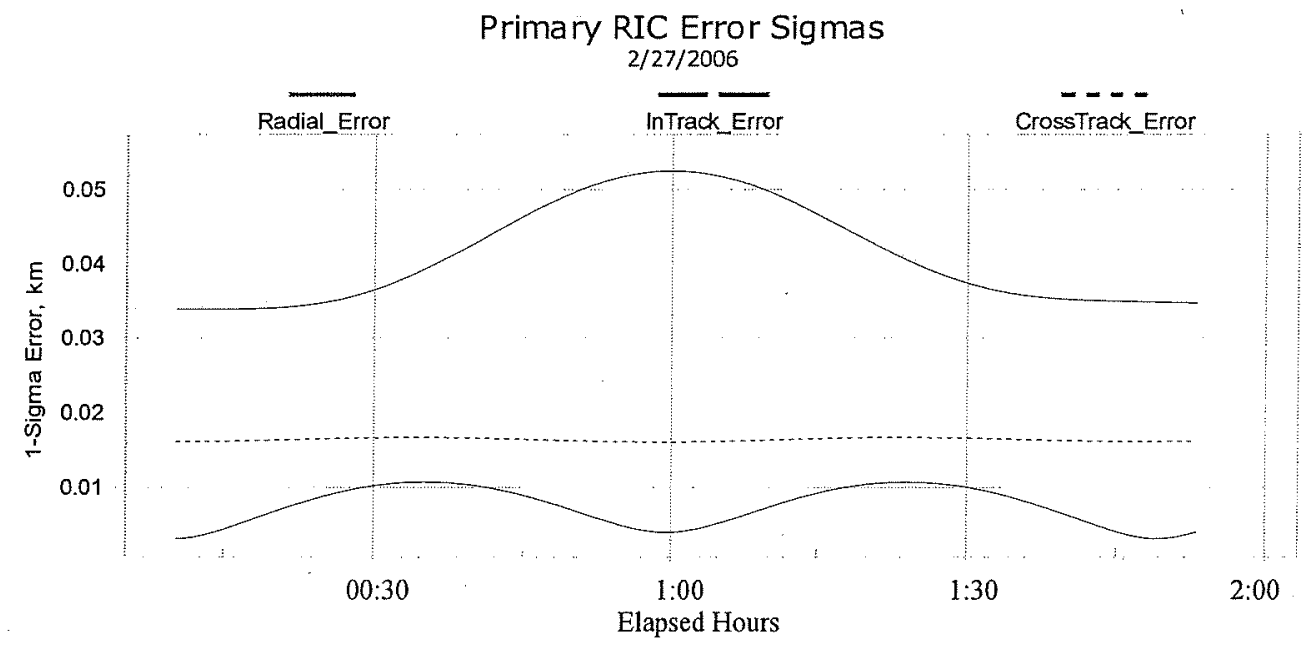

Figure 11. Primary object Covariance time history.

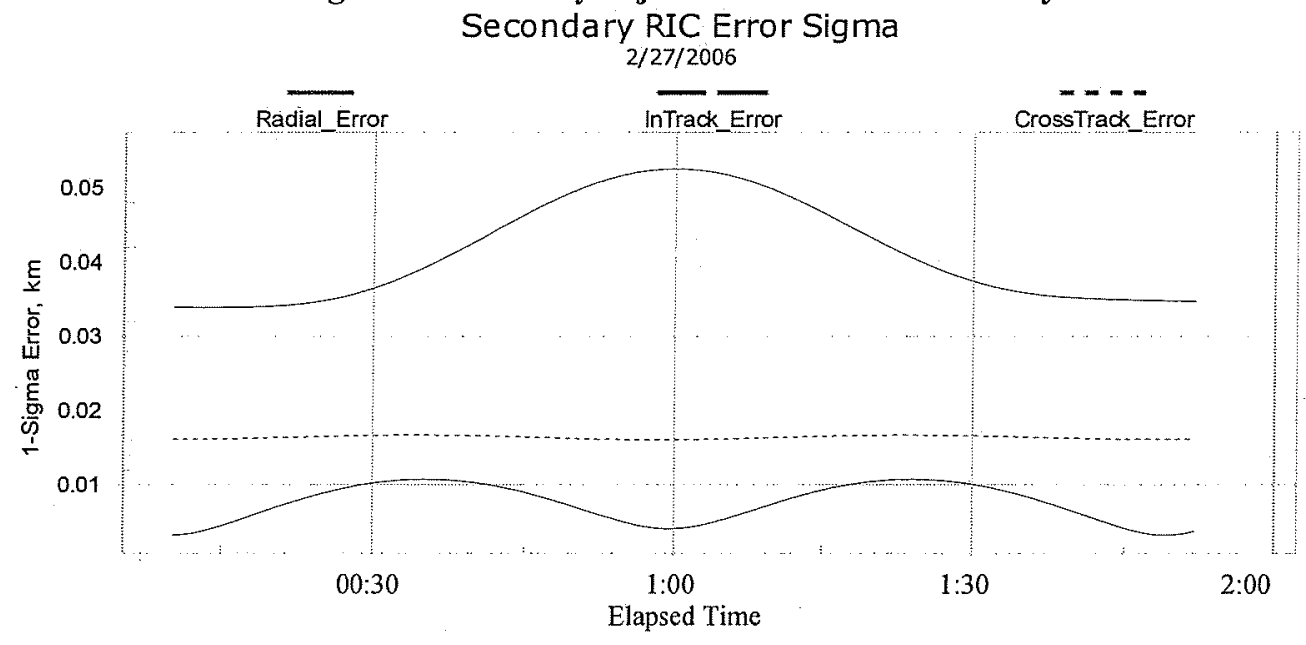

Figure 12. Secondary object Covariance time history. 


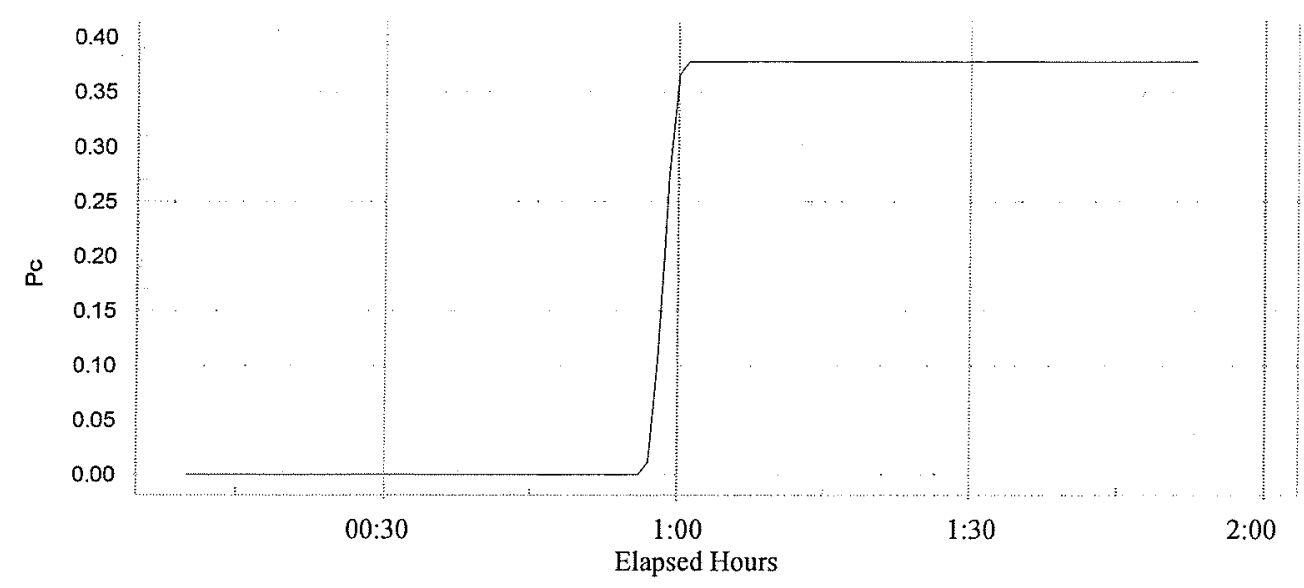

Figure 13. Probability of Collision time history.

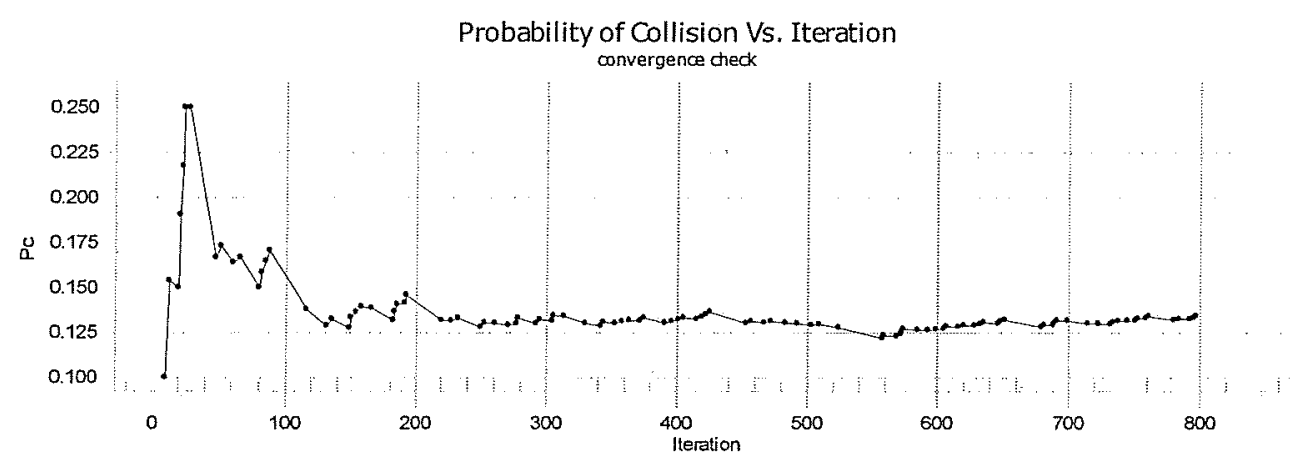

Figure 14. Probability of Collision versus iteration for the Monte Carlo simulation.

\section{Summary and Future Work}

An algorithm based on [6] has been implemented to calculate the probability of collision between spacecraft with nonlinear relative trajectories. The complex geometries that can be created in these encounters are broken down into simple geometries that are easily used as integration volumes. The resulting algorithm compares extremely well with existing 2D theory when analyzing linear relative trajectories. In addition, the new algorithm has also been shown to give comparable answers to a published reference solution for the case of a highly nonlinear circular relative trajectory and compares within an order of magnitude with Monte Carlo results.

The 3D tool is currently being used to analyze conjunctions with EOS and its fellow constellation members. While most conjunctions are high velocity, using the 3D tool allows the EOS Conjunction Assessment Analysts to begin gaining insight into the transition between linear and nonlinear relative trajectories. Additionally, should any low velocity encounters between constellation members or between EOS and other space objects occur, the tool is available to assess the collision risk.

\section{References}

1. Demarest, P. "The Impact of Maneuver Failures on the EOS Afternoon Constellation," (AIAA 02-4744), AIAA/AAS Astrodynamics Specialist Conference, Monterey, CA, August 2002.

2. Chan, K., "Collision Probability Analysis for Earth Orbiting Satellites," Advances in the Astronautical Sciences Vol. 96, pp.1033-1048, 1997. 
3. Alfriend, K.T., Akella, M. R., Lee, D., Frisbee, J., Foster, J. L., "Probability of Collision Error Analysis," Space Debris Vol. 1, No. 1, Kluwer Academic Publishers, p. 21-35, 1999.

4. Patera, R. P., "General Method for Calculating Satellite Collision Probability," Journal of Guidance, Control, and Dynamics, Vol. 24, No. 4, 2001.pp. 716-722.

5. Chan, K., "Spacecraft Collision Probability for Long-Term Encounters," American Astronautical Society, AAS Paper 03-549, Aug. 2003.

6. Patera, R., P., "Satellite Collision Probability for Non-Linear Relative Motion," Journal of Guidance, Control, and Dynamics, Vol. 26, No. 5, 2003. pp. 728-733.

7. Patera, R., P., "Collision Probability For Larger Bodies Having Non-Linear Relative Motion," American Astronautical Society, AAS Paper 05-309, Aug. 2005. 